\title{
Falha na primeira mamada em bezerros Guzerá: fatores predisponentes e parâmetros genéticos
}

\author{
Anita Schmidek 1,2 , Maria Eugênia Zerlotti Mercadante ${ }^{3}$, Mateus José Rodrigues Paranhos da \\ Costa $^{2,4,5}$, Alexander George Razook ${ }^{3,5}$, Leopoldo Andrade de Figueiredo 3 \\ ${ }^{1}$ Programa de Pós-Graduação em Genética e Melhoramento Animal, Departamento de Zootecnia, FCAV-UNESP, Jaboticabal, SP. Bolsista \\ CAPES. \\ 2 Grupo ETCO - Grupo de Estudos e Pesquisas em Etologia e Ecologia Animal. \\ ${ }^{3}$ Estação Experimental de Zootecnia de Sertãozinho - IZ/APTA/SAA-SP. \\ 4 Departamento de Zootecnia, FCAV-UNESP. \\ 5 Pesquisador CNPq.
}

RESUMO - Em decorrência da elevada ocorrência (18\%) de bezerros Guzerá que não mamaram por conta própria em suas mães (falha na primeira mamada), foram avaliados os fatores associados a estas falhas, sua influência na mortalidade e parâmetros genéticos para falha na primeira mamada (FPM). Dos 1.527 bezerros Guzerá nascidos de 1992 a 2004, 279 apresentaram FPM. Os efeitos fixos foram estudados utilizando o procedimento GENMOD-SAS com função de ligação probit. Parâmetros genéticos para FPM foram estimados por máxima verossimilhança restrita aproximada, ajustando modelo animal que incluiu efeitos genéticos diretos e maternos e de ambiente permanente da vaca. Verificou-se que os principais fatores predisponentes à FPM foram vacas com problema de úbere e tetos e bezerros pesando menos de $25 \mathrm{~kg}$ ao nascimento. FPM influenciou a mortalidade, com maior chance de óbitos para bezerros que falharam em comparação aos que mamaram naturalmente. A estimativa de herdabilidade do efeito materno foi baixa $(0,08)$ e a referente ao efeito direto, nula. A estimativa de repetibilidade também foi baixa $(0,17)$. Devido ao impacto de FPM na mortalidade de bezerros, sugere-se que a avaliação da conformação do aparelho mamário seja utilizada como um indicador para minimizar o problema. Entretanto, a baixa magnitude da estimativa de herdabilidade indica que progresso genético mediante seleção contra FPM poderia ser obtido somente a longo prazo.

Palavras-chave: aparelho mamário, falha de amamentação, bezerros de corte, herdabilidade

\section{Fail to suckle in Guzerá calves: underlying factors and genetic parameters}

\begin{abstract}
This study aimed to evaluate the effects related to the high occurrence (18\%) of Guzerá newborn calves that failed to suckle (FS) after birth. Data from 1527 births recorded from 1992 to 2004 were analyzed by a fixed effects model using the probit linking function with the GENMOD procedure of SAS ${ }^{\circledR}$. An animal model including direct and maternal additive genetic and cow permanent environment effects was also used to estimate genetic parameters for FS by quasi-restricted maximum likelihood. Cows with udder and teat problems and calves weighting less than $25 \mathrm{~kg}$ at birth were the major effects associated to the occurrence of FS in newborn calves which were more susceptible to mortality than those that successfully suckled. Maternal and direct effects heritability estimates for FS were respectively 0.08 and null. The repeatability estimate for FS was 0.17 . The evaluation of the conformation of the cow mammary system may be used as an indicator to minimize the occurrence of FS. Genetic progress by selection for reducing FS in Guzerá newborn calves may be expected only in the long term.
\end{abstract}

Key Words: mammary system, fail to suckle, beef calves, heritability

\section{Introdução}

Em sistemas de cria de bovinos de corte, a principal receita advém dos bezerros desmamados e, portanto, a mortalidade pré-desmama é o principal ponto de estrangulamento econômico (Bourdon \& Golden, 2000). Isto significa que não basta conceber ou mesmo parir um bezerro saudável se o mesmo não estiver vivo na desmama.
A mortalidade de bezerros é associada, muitas vezes, à imunização inadequada decorrente de atrasos ou falhas na primeira mamada. Na espécie bovina, a imunidade passiva depende exclusivamente da absorção intestinal de imunoglobulinas durante as primeiras horas após o nascimento, visto que a placenta dos animais ungulados dificulta a passagem intra-uterina de anticorpos (Brignole \& Stot, 1980). O ideal é que, em até três horas após o 
nascimento, o bezerro fique em pé, a vaca permita que o bezerro tenha acesso aos tetos e o bezerro consiga mamar de modo que obtenha o colostro através da amamentação (Schmidek et al., 2006).

Entretanto, apesar de ser um comportamento necessário à sobrevivência, nem todos os bezerros nascidos mamam. Em raças bovinas com aptidão leiteira, são relatados índices de falha de amamentação em torno de $30 \%$, decorrentes de aspectos como conformação do aparelho mamário da vaca, idade da vaca ao parto, vigor do bezerro, peso ao nascer, bem como do comportamento da vaca e do bezerro (Edwards, 1982; Frisch, 1982). Se na pecuária leiteira falhas na primeira mamada são facilmente contornáveis, visto que há contato diário de tratadores com os animais, com predominância de aleitamento artificial, na pecuária de corte, que usualmente é conduzida de forma extensiva e com mínima intervenção humana, falhas na primeira mamada representam elevação de custos com mão-de-obra e/ou elevação nos índices de mortalidade pré-desmama. Em raças com histórico de seleção para dupla aptidão (carne e leite), como é o caso da raça Guzerá, é esperada maior ocorrência de falha de amamentação em comparação a raças de corte, nas quais problemas deste tipo parecem ser mais raros (Edwards \& Broom, 1982; Paranhos da Costa et al., 1996).

Este estudo foi realizado com os objetivos de buscar melhor entendimento sobre os fatores causais que resultam em falhas na primeira mamada e encontrar soluções para a redução dos problemas de falhas na primeira mamada de bezerros da raça Guzerá.

\section{Material e Métodos}

Este estudo foi conduzido com dados de 1.527 bezerros Guzerá, nascidos entre 1992 e 2004 na Estação Experimental de Zootecnia de Sertãozinho (EEZS). Esta unidade experimental do Instituto de Zootecnia está localizada ao norte do estado de São Paulo (latitude $21^{\circ} 102^{\prime}$ sul e longitude $48^{\circ} 52^{\prime}$ oeste), região que apresenta clima tropical úmido, com temperatura e precipitação anual média de $24^{\circ} \mathrm{C}$ e $1.312 \mathrm{~mm}$, respectivamente. Os pastos são formados principalmente por Panicum maximum e Brachiaria brizantha.

O rebanho Guzerá da EEZS é formado por animais puros de origem e desde 1980 são utilizados somente reprodutores do próprio plantel, excetuando-se a introdução de 22 vacas provenientes de rebanho leiteiro em 1995 e 1996 e a utilização de um touro proveniente de outro rebanho em 1995. A seleção dos touros (10\% dos machos avaliados) é baseada no maior diferencial para peso padronizado aos 378 dias de idade, obtido ao final de teste de desempenho em confinamento de 168 dias. Os touros são utilizados por, no máximo, duas estações reprodutivas (touros com dois ou três anos de idade).

A seleção das matrizes é baseada no maior diferencial para peso padronizado aos 550 dias de idade, obtido em pastagem, sendo retidas 50-60\% das novilhas avaliadas. As matrizes são descartadas por falha reprodutiva em dois anos consecutivos ou por idade avançada, quando acima de 11 anos. Eventualmente, foram também descartadas vacas que apresentaram problemas de saúde, de habilidade materna ou de temperamento. A estação de monta natural ocorre entre novembro e fevereiro, resultando em nascimentos entre os meses de setembro a dezembro. Maiores detalhes do rebanho e do processo seletivo estão descritos em Mercadante et al. (2004).

No dia seguinte à parição, vacas e bezerros são pesados, identificados e conduzidos aos pastos onde permanecem até a desmama, que ocorre por volta dos sete meses de idade. Entre o nascimento e a desmama, vacas e bezerros são trazidos três vezes ao curral, correspondendo aos procedimentos de formação dos lotes de monta, final da estação de monta e desmama. Os bezerros foram pesados no final da estação de monta e na desmama.

Os bezerros avaliados eram filhos de 75 touros, com progênies variando de 2 a 46 bezerros, e de 535 vacas, com variação de 1 a 10 filhos por vaca. Para a análise genética, foi montada matriz de parentesco com 2.306 animais, retrocedendo até 10 gerações a partir dos bezerros analisados.

A partir de 1992, durante o manejo de identificação e pesagem dos bezerros, iniciou-se o registro de bezerros que não haviam mamado - fato avaliado pelo aparelho mamário (úbere e tetos) da vaca com sinais de que tinha sido mamado ou não e pelo bezerro com barriga cheia ou vazia. Para bezerros que não haviam mamado, a vaca era contida no tronco e o bezerro induzido a mamar na vaca, com auxílio dos vaqueiros. Foi considerada falha na primeira mamada (FPM) quando o bezerro recebeu ajuda para mamar - característica codificada como 1 (houve FPM) ou 0 (não houve FPM), sendo excluídos das análises bezerros natimortos e gêmeos. Foi avaliada também a mortalidade do bezerro até a pesagem do final da estação de monta, quando tinham em média quatro meses de idade, sendo 1 (bezerros vivos na pesagem) ou 0 (bezerros que morreram entre o nascimento e a pesagem).

Todos os efeitos fixos que poderiam ser usados como covariáveis (idade e altura da vaca ao parto, peso ao nascer e endogamia do bezerro) foram estudados em classes, em razão de o modelo ter apresentado melhor ajuste. Além disso, considerar os efeitos fixos em classes permitiu comparações dentro de cada efeito. 
O aparelho mamário foi analisado com duas classes ( 1 = com problema, incluindo vacas com registro de úberes pendulosos, tetos grossos, grandes ou perdidos; $0=$ sem problema), a idade da vaca ao parto com três classes ( $\leq$ quatro anos, referente a vacas jovens; cinco a oito anos, referente a vacas adultas; $\geq$ nove anos, referente a vacas velhas) e a altura da vaca ao parto com cinco classes, elaboradas de acordo com a média e o desvio-padrão da característica $\leqslant 1,33 \mathrm{~m} ; 1,34$ a $1,37 \mathrm{~m} ; 1,38$ a $1,41 \mathrm{~m}$; $1,42$ a $1,45 \mathrm{~m} ; \geq 1,46 \mathrm{~m})$.

Os efeitos fixos relativos aos bezerros compreenderam 13 anos de nascimento (1992 a 2004), cinco meses de nascimento agrupados naqueles de maior concentração (setembro; outubro; novembro), peso ao nascer, analisado em cinco classes, também elaboradas de acordo com a média e o desvio-padrão da característica $(\leq 21 \mathrm{~kg} ; 22$ a $25 \mathrm{~kg} ; 26$ a $30 \mathrm{~kg} ; 31$ a $34 \mathrm{~kg} ; \geq 35 \mathrm{~kg}$ ), e o coeficiente de endogamia, analisado em quatro classes ( $0 \%$; >0 a $4 \%$; >4 a $8 \%$; >8\%).

A falha na primeira mamada (FPM) e a mortalidade foram analisadas utilizando o procedimento GENMOD (SAS, 2001), assumindo distribuição binomial dos dados, com função de ligação probit a uma distribuição adjacente normal. A probabilidade de FPM = 1 (e mortalidade = 1) é dada por $\phi^{-1}$ (taxa de FPM), em que fé a função de distribuiçãonormal acumulada. Além dos efeitos principais, foram testadas também todas as interações duplas, as quais foram nãosignificativas $(\mathrm{P}>0,05)$.

Razões entre as chances (odds ratios) foram calculadas de modo a obter informação sobre o risco relativo da ocorrência de falha de amamentação ou de mortalidade, entre as classes dos efeitos fixos que foram significativos $(\mathrm{P}<0,05)$. Para um efeito fixo com duas classes, razão de chances igual a 1 indica ausência de diferença entre as duas classes, ou seja, as duas têm a mesma chance de ocorrência, e um intervalo de confiança de $95 \%$ que contenha 1 indica que a razão de chance, em relação a uma classe específica, não é estatisticamente diferente (SAS, 2001). As razões entre as chances foram construídas em relação à classe com menor ocorrência do problema (FPM e mortalidade).

Os componentes de variância foram estimados por máxima verossimilhança restrita aproximada usando o programa ASReml (Gilmour et al., 1999). Foram considerados quatro modelos, incluindo os efeitos aleatórios: 1) genético aditivo direto do bezerro, genético aditivo materno e de ambiente permanente da vaca; 2) genético aditivo direto do bezerro e genético aditivo materno; 3$)$ genético aditivo direto do bezerro e de ambiente permanente da vaca e 4) genético aditivo direto e de ambiente permanente da vaca. Todos os modelos incluíram também os efeitos fixos significativos $(\mathrm{P}<0,05)$ e o efeito aleatório residual.
A variância residual foi fixada em uma unidade da medida, $\sigma^{2}=1$. Os parâmetros genéticos foram estimados após convergência, que foi assumida quando o valor do logaritmo da função de verossimilhança mudou menos que 0,002 entre duas iterações.

\section{Resultados e Discussão}

As incidências para as falhas na primeira mamada (FPM) e mortalidade até quatro meses de idade foram 18 e $10 \%$, respectivamente (Tabela 1 ).

$\mathrm{O}$ ano de nascimento do bezerro influenciou $\operatorname{FPM}(\mathrm{P}<0,01)$, mas não a mortalidade até quatro meses. De acordo com as médias não-ajustadas e as razões de chance (não mostradas), houve tendência descrecente de ocorrência de FPM entre 1992 e 1997 e estabilização após 1998. Entretanto, apesar da aparente redução de FPM ao longo dos anos, não houve ano com ausência ou ocorrência reduzida do problema (Figura 1). A tendência de redução do problema pode ser decorrente do procedimento de descartar vacas cujos bezerros repetidamente apresentaram falhas na primeira mamada. Porém, após mais de dez anos, o índice de ocorrência do problema ainda é elevado, indicando a necessidade de outra estratégia visando minimizar o problema.

A mortalidade parece estar associada à ajuda para o bezerro mamar, diminuindo nos anos com menor FPM e aumentando em anos com maior FPM, porém com tendência constante ao longo dos anos avaliados. Especificamente em 1997, o procedimento de ajudar o bezerro a mamar foi restringido, a fim de minimizar a mão-de-obra, o que resultou em menor incidência de FPM e, conseqüentemente, maior mortalidade. Deve ser ressaltado que o menor registro de FPM neste ano não significa que a freqüência de bezerros

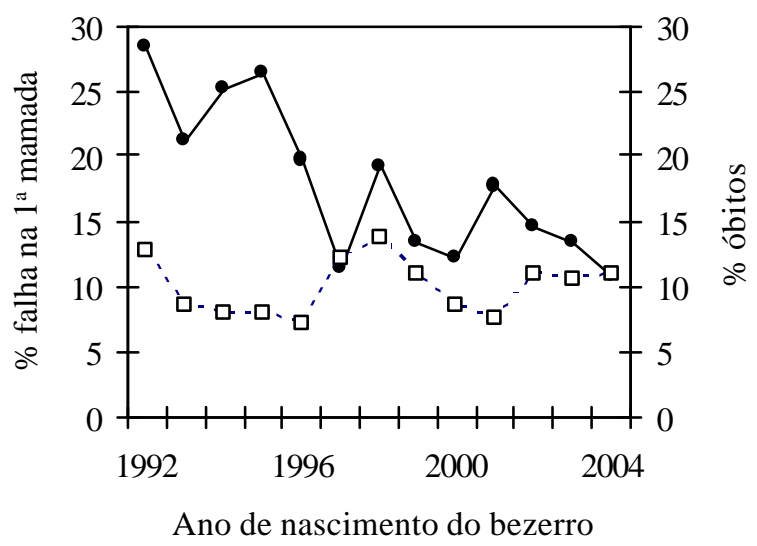

Figura 1 - Porcentagem de falhas na primeira mamada (linha contínua) e mortalidade (linha tracejada), em função do ano de nascimento do bezerro. 
Tabela 1 - Falhas na primeira mamada (FPM) e mortalidade em bezerros Guzerá, em função das classes de efeitos fixos

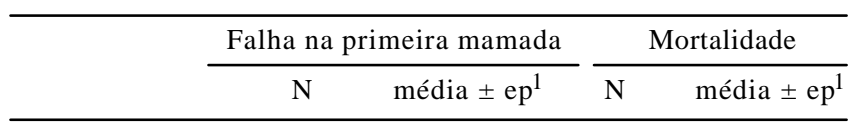

Aparelho mamário

Sem problema

1.388

$0,13 \pm 0,01 \quad 1.376$

$0,67 \pm 0,04 \quad 136$

$0,10 \pm 0,01$

Com problema

139

$0,15 \pm 0,03$

Idade da vaca ao parto

$\begin{array}{ll}3 \text { a } 4 \text { anos } & 503 \\ 5 \text { a } 8 \text { anos } & 713\end{array}$

\section{$0,10 \pm 0,01 \quad 497$}

$0,20 \pm 0,02 \quad 709$

$0,29 \pm 0,03 \quad 306$

$0,11 \pm 0,01$

$0,09 \pm 0,01$

$0,12 \pm 0,02$

Altura da vaca ao parto

$\leq 1,33 \mathrm{~m}$

$1,34-1,37 \mathrm{~m} \quad 326$

$1,38-1,41 \mathrm{~m} \quad 698$

$1,42-1,45 \mathrm{~m} \quad 373$

$\geq 1,46 \mathrm{~m}$

60

$0,10 \pm 0,04$

$0,16 \pm 0,02$

70

$0,17 \pm 0,01 \quad 692$

$0,23 \pm 0,02 \quad 368$

$0,32 \pm 0,06 \quad 59$

$0,06 \pm 0,03$

$0,09 \pm 0,02$

$0,12 \pm 0,01$

$0,08 \pm 0,01$

$0,14 \pm 0,05$

Peso ao nascer

$\leq 21 \mathrm{~kg}$

22-25 kg

26-30 kg

31-34 kg

$\geq 35 \mathrm{~kg}$

75

336

$0,39 \pm 0,06$

$0,26 \pm 0,02$

72

$742 \quad 0,17 \pm 0,01 \quad 736$

$279 \quad 0,11 \pm 0,02 \quad 278$

95

$0,11 \pm 0,03$

93

$0,14 \pm 0,04$

$0,14 \pm 0,02$

$0,09 \pm 0,01$

$0,10 \pm 0,02$

$0,08 \pm 0,03$

Sexo

Macho

$0,20 \pm 0,02 \quad 759$

$0,11 \pm 0,01$

Fêmea

762

$0,16 \pm 0,01$

753

$0,09 \pm 0,01$

Falhas na primeira mamada

\begin{tabular}{lcccc} 
Sem falha & - & - & 1.236 & $0,09 \pm 0,01$ \\
Com falha & - & - & 276 & $0,15 \pm 0,02$ \\
\hline Total & 1.527 & $0,18 \pm 0,01$ & 1.512 & $0,10 \pm 0,01$ \\
\hline
\end{tabular}

${ }^{1}$ número de observações; ${ }^{2}$ média observada terro-padrão da média.

que não mamaram no momento do manejo tenha sido também inferior. Provavelmente, a ocorrência de FPM foi semelhante aos outros anos avaliados.

Entre os efeitos estudados, peso do bezerro ao nascer e problema de aparelho mamário foram os de maior importância para FPM $(\mathrm{P}<0,01)$, em razão dos maiores valores do risco relativo (Tabela 2 ).

Neste estudo ficou evidente que aparelhos mamários com problema (freqüentemente úbere e tetos muito grandes ou, ocasionalmente, tetos perdidos) estão associados a falhas na primeira mamada (FPM) dos bezerros, com risco relativo 4,26 vezes maior $(\mathrm{P}<0,01)$ de haver falha em casos de aparelhos mamários com problema em comparação aos sem problema (Tabela 2). Em estudo abordando a importância da conformação do aparelho mamário (AM) para a amamentação de bezerros recém-nascidos, Ventrop \& Michanek (1992) destacaram que, em geral, bezerros filhos de vacas com AM grande não conseguiram mamar naturalmente em suas mães, precisando ser ajudados a

Tabela 2 - Risco relativo e intervalo de confiança a 95\% de falha de amamentação em bezerros Guzerá, de acordo com as classes de efeitos fixos

\begin{tabular}{|c|c|c|}
\hline \multirow[t]{2}{*}{ Item } & \multicolumn{2}{|c|}{ Falha de amamentação } \\
\hline & Risco relativo $\pm \mathrm{ep}^{1}$ & Intervalo de confiança \\
\hline
\end{tabular}

Aparelho mamário

sem problema

com problema

CR

$4,26 \pm 0,54$

CR

$1,36 \pm 0,14$

$1,71 \pm 0,21$

9 anos ou mais
5 a 8 anos

CR

$3,31-5,47 * *$

CR

$1,11-1,68 * *$

$1,35-2,18 * *$

Altura da vaca ao parto

$\leq 1,33 \mathrm{~m}$

1,34-1,37 m

1,38-1,41 m

$1,42-1,45 \mathrm{~m}$

$\geq 1,46 \mathrm{~m}$

CR

$1,27 \pm 0,32$

$1,35 \pm 0,33$

$1,87 \pm 0,47$

$1,97 \pm 0,62$

$\mathrm{CR}$

0,77-2,08

$0,84-2,18$

1,14-3,06*

$1,06-3,66^{*}$

Peso ao nascer

$\leq 21 \mathrm{~kg}$

$22-25 \mathrm{~kg}$

26-30 kg

31-34 kg

$\geq 35 \mathrm{~kg}$

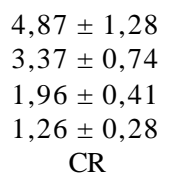

$2,91-8,16 * *$

$2,19-5,18 * *$

$1,31-2,94 * *$

0,81-1,94

CR

Sexo

macho

fêmea

$1,52 \pm 0,14$

CR

1,27-1,81**

$\mathrm{CR}$

${ }^{1}$ risco relativo \pm erro-padrão; $C R=$ classe referência; ${ }^{*}=P<0,05 ;{ }^{* *}=P<0,01$.

mamar ou receber leite de mamadeira (caso contrário, morreriam de fome).

O elevado risco de ocorrência de FPM nos casos de problemas de AM indica que o monitoramento do AM ao parto é importante para a raça Guzerá e que a conformação do AM pode ser utilizada como característica indicadora do problema de amamentação dos bezerros. Assim, espera-se redução na falha de amamentação com o descarte de vacas que repetidamente apresentam problemas de AM.

Ainda considerando os efeitos relativos às vacas, a idade destas ao parto foi o segundo em ordem de importância $(\mathrm{P}<0,01)$, com maior risco relativo de FPM em vacas acima de nove anos, bem como em vacas de cinco a nove anos, em comparação a vacas abaixo de quatro anos, resultado que era esperado. Frisch (1982) também reportou maior falha de amamentação em bezerros filhos de vacas mais velhas, decorrente da maior incidência de problemas de AM na classe de vacas de cinco anos ou mais em comparação a vacas de quatro anos ou menos. Neste estudo, a interação idade da vaca $\times$ AM não foi significativa $(\mathrm{P}>0,05)$, provavelmente em decorrência da estrutura dos dados, em que a grande maioria das vacas não apresentou problemas de 


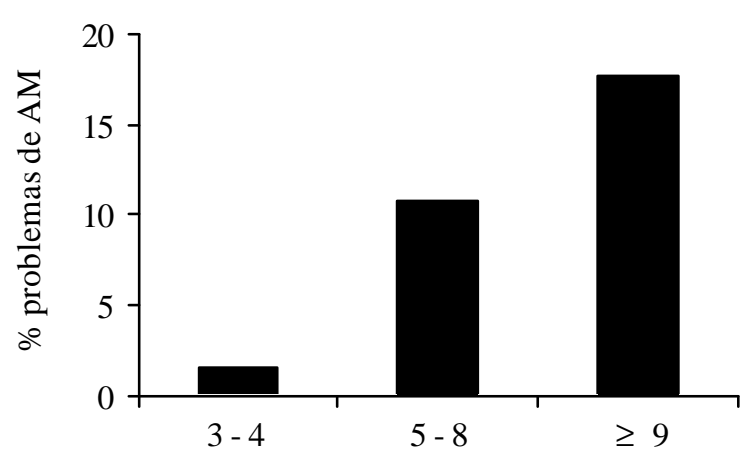

Idade da vaca ao parto (anos)

Figura 2 - Percentual de vacas com problema de aparelho mamário (AM) em cada classe de idade ao parto.

AM, mesmo na classe de vacas mais velhas $(82,3 \%$ sem problema de AM). Por outro lado, se forem consideradas apenas as vacas com problema de AM (Figura 2), observa-se tendência de aumento dos problemas de AM com o avançar da idade, o que é explicado pela ocorrência de relaxamento e distensão dos tecidos do AM com o aumento da idade da vaca. Porém, destaca-se a ocorrência de problemas de AM na classe de vacas jovens, ainda que de forma reduzida.

A classe de altura da vaca ao parto também influenciou a ocorrência de FPM $(\mathrm{P}<0,01)$, porém nem todas as classes diferiram entre si. A relação entre FPM e a classe de altura da vaca ao parto (AP) apresentou resultado oposto ao esperado, com maior ocorrência de FPM para vacas mais altas em comparação às mais baixas $(\mathrm{P}<0,05)$. Sabendo que os tetos da vaca devem estar em altura semelhante à da cabeça do bezerro, pois estes raramente ajoelham para mamar, e que a ocorrência de vacas com tetos muito altos ou pequenos, difcultando o acesso aos bezerros, foi nula, esperava-se maior problema de amamentação para bezerros filhos de vacas mais baixas, prejudicando a amamentação. Ventrop \& Michanek (1992) relataram ocorrência de FPM para AM próximo ao solo, especialmente com distância entre o úbere e o solo inferior a $46 \mathrm{~cm}$.

A altura da vaca pode estar associada a outra característica, como a idade da vaca ao parto, com maior proporção de vacas mais velhas na classe de vacas mais altas, para os casos de ocorrência de FPM. Ou seja, das vacas com cinco anos ou mais, cujos bezerros apresentaram FPM, 80,6\% apresentaram altura superior ou igual a 1,43 m. Por outro lado, entre as vacas com cinco anos ou mais, cujos bezerros não apresentaram FPM, 57,5\% apresentaram altura superior ou igual a 1,43 m. Estes resultados indicam a necessidade de mensurações mais específicas, a exemplo das utilizadas por Ventrop \& Michanek (1992), mensurando a distância entre o AM e o solo.

Considerando os efeitos estudados, o peso ao nascer do bezerro foi de grande importância para a ocorrência de FPM. Pesos inferiores a $25 \mathrm{~kg}$ e especialmente inferiores a $21 \mathrm{~kg}$ apresentaram os maiores riscos relativos $(\mathrm{P}<0,01)$.

Bezerros com pesos ao nascer (PN) de $25 \mathrm{~kg}$ ou menos representaram 19,0\% dos nascimentos avaliados e apresentaram risco relativo de FPM aproximadamente quatro vezes maior $(\mathrm{P}<0,01)$ em comparação aos que pesaram $35 \mathrm{~kg}$ ou mais (Tabela 2). O impacto de baixos PN na mortalidade de bezerros é amplamente aceito, a exemplo da pesquisa de Azzam et al. (1993), que relataram maior mortalidade para bezerros pequenos considerando seu grupo genético, sexo e idade da vaca ao parto. Usualmente, a maior mortalidade de bezerros muito leves está associada ao baixo vigor destes. Neste estudo, além do problema de vigor, baixo PN pode estar associado à ausência de leite no estômago de bezerros que não mamaram, em comparação à presença de leite dos que mamaram, uma vez que a ajuda para mamar ocorreu após a pesagem dos mesmos. Ou seja, não está claro que baixo PN seja causa ou consequiência da ocorrência de FPM. Frisch (1982) relatou tendência $(\mathrm{P}<0,10)$ de menor $\mathrm{PN}$ para bezerros filhos de vacas com problemas de AM nos quatro tetos $(\mathrm{PN}=26,90 \mathrm{~kg}$ ) em comparação a vacas sem problemas de AM $(\mathrm{PN}=29,90 \mathrm{~kg})$, diferença decorrente de falha de amamentação.

$O$ risco relativo de machos apresentarem FPM foi 1,52 vezes maior em comparação às fêmeas $(\mathrm{P}<0,01)$. Não foi encontrado estudo tratando especificamente deste aspecto, porém Machado Neto et al. (1997), avaliando o mesmo rebanho deste estudo, encontraram menor concentração de imunoglobulinas em machos, resultados que possivelmente estejam associados, pois, havendo maior falha de amamentação em machos, é esperado que esta categoria apresente também menor concentração de imunoglobulinas. Estudos avaliando outras características ligadas à sobrevivência do bezerro freqüentemente relatam resultado desfavorável para os machos em comparação às fêmeas. Abordando o vigor de bezerros Brahman, Riley et al. (2004) reportaram risco relativo 1,44 vezes maior de machos apresentarem baixo vigor ao nascimento em comparação às fêmeas. Em estudo sobre mortalidade de bezerros da raça Pardo Suíço, Erf et al. (1990) também relataram risco relativo de óbitos 1,5 vezes maior para machos do que para fêmeas. O resultado obtido possivelmente seja decorrente de estratégia evolutiva, considerando que, para a perpetuação da espécie em questão, seja mais importante haver maior número de fêmeas que de machos na população. 
Para a mortalidade, falha de amamentação foi o único efeito significativo $(\mathrm{P}<0,05)$. O risco de bezerros que falharam em mamar morrerem foi 1,28 vezes o risco de bezerros que mamaram morrerem.

O maior risco relativo de óbitos em bezerros que falharam em mamar era esperado, pois é conhecido que em bovinos a aquisição de imunidade passiva durante a fase de gestação é desprezível, e que esta depende da absorção intestinal de imunoglobulinas provenientes do colostro materno, que deve ocorrer mediante amamentação durante as primeiras horas após o nascimento (Cunningham, 1993; Machado Neto et al., 1997). Avaliando o comportamento de bezerros Guzerá e Nelore pertencentes ao rebanho do presente estudo, Schmidek et al. (2006) identificaram maior mortalidade $(\mathrm{P}<0,05)$ para bezerros que não mamaram em até três horas após o nascimento $(17,5 \%)$ em comparação aos que mamaram em até três horas de vida $(4,0 \%)$.

Entretanto, apesar da maior mortalidade nos casos de FPM, deve ser destacado que o procedimento de ajudar o bezerro a mamar, do ponto de vista econômico e de bemestar animal, foi muito eficiente, salvando cerca de $85 \%$ desses bezerros, que, de outra forma, provavelmente teriam morrido de fome. Assim, se forem consideradas condições de criação totalmente extensivas, em que o procedimento de ajudar o bezerro a mamar não seria realizado, as ocorrências de FPM resultariam em óbito de grande parte destes bezerros, e a mortalidade pré-desmama poderia atingir $25,6 \%$.

Em todos os modelos em que o efeito genético aditivo direto (do bezerro) foi incluído, o programa, por ação padrão, limitou o componente de variância a um valor muito baixo, mas positivo (Gilmour et al., 1999), indicando que, nestes casos, a estimativa da variância genética aditiva direta e a herdabilidade do efeito direto é nula (Tabela 3). Por outro lado, ao não incluir o componente genético aditivo direto (Modelo 4), as estimativas dos efeitos maternos, tanto genético como de ambiente permanente, foram muito próximas das estimadas nos modelos anteriores, indicando que o componente genético aditivo direto realmente é nulo, e a característica FPM é essencialmente materna. No Modelo 2, em que o efeito do ambiente permanente matemo não foi considerado, a variância genética aditiva materna e a herdabilidade materna foi superestimada. Como os 1.527 bezerros são filhos de 535 vacas (média de 2,9 bezerros por vaca), o efeito de ambiente permanente pode ser estimado com relativa acurácia e deve ser incluído no modelo para não inflacionar o efeito genético materno. Assim, o Modelo 4 pode ser considerado o mais adequado para FPM, com estimativas do componente de variância, em razão de a mãe ter explicado $17 \%$ da variação fenotípica. O parâmetro escala $(\phi)$ é freqüentemente usado para indicar a dispersão dos dados, e um $\phi=0,63$ indica subdispersão, embora possa também indicar outros problemas como presença de outliers ou um modelo incorretamente especificado (Gilmour et al., 1999). Entretanto, vale ressaltar que os dados analisados foram previamente checados quanto à presença de informações discrepantes e que o uso da função de ligação probit foi embasado em estudos anteriores abordando características de limiar (McGuirk et al., 1998; Kadarmideen et al., 2000).

A estimativa de herdabilidade da FPM $(0,08 \pm 0,07)$ desencoraja a seleção como meio de reduzir este problema. Não foram encontradas referências sobre o assunto; entretanto, o fato de o ambiente permanente da vaca ter sido o componente de variância mais importante evidencia que alguma característica das vacas, possivelmente a conformação do AM, tenha mais importância na ocorrência de FPM do que componentes genéticos aditivos.

Considerando o fato de que o peso ao nascer (PN) foi um efeito importante na ocorrência de FPM e que estimativas de herdabilidade direta (bezerro) de PN são conhecidamente moderadas a altas (Mercadante et al., 1995; Cyrillo et al., 2004), não era de se esperar que o componente genético direto de FPM fosse desprezível. Possivelmente, este resultado seja decorrente de um confundimento, já descrito acima, referente a bezerros leves que eram realmente pequenos e bezerros nascidos com peso regular, mas que não mamaram e, por isso, apresentaram peso reduzido.

A baixa estimativa de repetibilidade da característica FPM $(0,17 \pm 0,05)$ pode decorrer do fato de que tanto características da vaca como do bezerro tenham importância na ocorrência do problema, indicando que não é recomendado

Tabela 3 - Componentes de variância e parâmetros genéticos para falha de amamentação em bezerros Guzerá

\begin{tabular}{lcccccccc}
\hline Modelo & $\operatorname{Var}(\mathrm{a})^{1}$ & $\operatorname{Var}(\mathrm{m})$ & $\operatorname{Var}(\mathrm{c})$ & $\operatorname{Var}(\mathrm{p})$ & $\phi$ & $\mathrm{h}^{2}$ & $\mathrm{~m}^{2}$ & $\mathrm{r}$ \\
\hline 1 & $0,65^{-8 \mathrm{~L}}$ & 0,09 & 0,12 & 1,21 & 0,63 & $0,00 \pm 0,00$ & $0,08 \pm 0,07$ & $0,17 \pm 0,05$ \\
2 & $0,19^{-8 \mathrm{~L}}$ & 0,20 & - & 1,20 & 0,65 & $0,00 \pm 0,00$ & $0,18 \pm 0,05$ & - \\
3 & $0,11^{-8 \mathrm{~L}}$ & - & 0,20 & 1,20 & 0,63 & $0,00 \pm 0,00$ & - & $0,16 \pm 0,05$ \\
4 & - & 0,09 & 0,12 & 1,21 & 0,63 & - & $0,08 \pm 0,07$ & $0,17 \pm 0,05$ \\
\hline
\end{tabular}

${ }^{1}$ variância genética aditiva direta; variância genética aditiva materna; variância de ambiente permanente; variância fenotípica; parâmetro escala; herdabilidade direta; herdabilidade materna; repetibilidade; Llimitado 
descartar vacas na primeira ocorrência do problema, devendo-se esperar que o problema se repita.

Foram registradas 116 vacas que voltaram a parir após seus bezerros apresentarem FPM, somando 379 partos entre a primeira ocorrência do problema e a saída da vaca do rebanho. Em 61,6\% destes partos houve FPM. Por outro lado, se do total de 116 vacas forem consideradas somente as 23 que apresentaram problema de aparelho mamário (52 partos), constata-se que em $93,8 \%$ dos partos houve FPM. Estes resultados indicam maior risco da repetição de FPM quando a causa foi relacionada a problema de aparelho mamário da vaca, sendo recomendado registrar se a falha de amamentação ocorreu devido a problemas da vaca ou do bezerro, de modo a otimizar o descarte de vacas cujos bezerros apresentaram FPM.

O estudo de problemas de aparelho mamário deve ser conduzido neste rebanho, visando melhor entendimento da ocorrência de problemas de aparelho mamário, bem como suas causas, fornecendo subsídios para minimizar, de forma indireta, a ocorrência de falhas na primeira mamada.

\section{Conclusões}

Devido à importância do aparelho mamário na ocorrência de falha na primeira mamada, a conformação deste deve ser utilizada como medida indireta do problema, esperando-se redução na falha de amamentação e, conseqüentemente, na mortalidade, com o descarte de vacas que repetidamente apresentem problemas de aparelho mamário.

A baixa herdabilidade para falha na primeira mamada indica que a seleção contra este problema pode resultar em pequeno progresso genético, demandando longo prazo.

\section{Agradecimento}

À pesquisadora Joslaine N. S. G. Cyrillo, pelo incentivo ao estudo destes dados e auxílio na fase inicial das análises, e também à colaboração dos assistentes de pesquisa José Furtado e José Valdir Trevisan, que auxiliaram na parte prática de auxílio para mamar e nos registros de campo destas informações.

\section{Literatura Citada}

AZZAM, S.M.; KINDER, J.K.; NIELSEN, L.A. et al. Environmental effects on neonatal mortality of beef calves. Journal of Animal Science, v.71, p.282-290, 1993.

BOURDON, R.; GOLDEN, B. [2000]. EPD and economics determining the relative importance of traits. Disponível em $><$ http://beef-mag.com/mag/beef_epds_economics_determining/ index.html. Acesso em: 18/12/2006.
BRINGNOLE, T.J.; STOTT, G.H. Effect of suckling followed by bottle feeding colostrum on immunoglobulin absorption and calf survival. Journal of Dairy Science, v.63, p.451456,1980 .

CUNNINGHAM, J.G. Tratado de fisiologia veterinária. 1.ed. Rio de Janeiro: Guanabara Koogan, 1993. 454p.

CYRILLO, J.N.S.G.; ALENCAR, M.M.; RAZOOK, A.G. et al. Modelagem e estimação de parâmetros genéticos e fenotípicos para pesos do nascimento ao momento da seleção (378 dias) de machos Nelore. Revista Brasileira de Zootecnia, v.33, n.6, p.1405-1415, 2004.

EDWARDS, S.A. Factors affecting the time to first suckling in dairy calves. Animal Production, v.34, p.339-346, 1982.

EDWARDS, S.A.; BROOM, D. Behavioural interactions of dairv cows with their newborn calves and the effects of parity. Animal Behavior, v.30, p.525-535, 1982.

ERF, D.F.; HANSEN, L.B.; NIETZEL, R.R. Inheritance of calf mortality for Brown Swiss cattle. Journal of Dairy Science, v.73, n.4, p.1130-1134, 1990.

FRISH, J.E. The use of teat-size measurements or calf weaning weight as an aid to selection against teat defects in cattle. Animal Production, v.32, p.127-133, 1982.

GILMOUR, A.R.; CULLIS, B.R.; WELHAM, S.J. et al. ASReml Reference Manual. Orange: NSW Agriculture, 1999. 181p.

KADARMIDEEN, H.N.; THOMPSON, R.; SIMM, G. Linear and threshold model genetic parameters for disease, fertility and milk production in dairy cattle. Animal Science, v.71, p.411419, 2000.

MACHADO NETO, R.; PACKER, I.U.; BONILHA, L.M. et al. Concentração de $\operatorname{IgG}$ sérica em bezerros das raças Nelore, Guzerá, Gir e Caracu. 1. Estatísticas descritivas e causas de variação. Revista Brasileira Zootecnia, v.26, n.5, p.914919, 1997.

MCGUIRK, B.J.; GOING, I.; GILMOUR, A.R. The genetic evaluation of beef sires used for crossing with dairy cows in the UK. 2. Genetic parameters and sire merit predictions for calving survey traits. Animal Science, v.66, p.47-54, 1998.

MERCADANTE, M.E.Z.; LÔBO, R.B.; BORJAS, A.R. Parámetros genéticos para características de crecimiento en cebuínos de carne. Archivos Latinoamericanos de Producción Animal, v.3, p.45-89, 1995.

MERCADANTE, M.E.Z.; RAZOOK, A.G.; CYRILLO, J.N.S.G. et al. Programa de Seleção na Estação Experimental de Zootecnia de Sertãozinho: resultados de pesquisas, sumário de touros Nelore. Nova Odessa: Instituto de Zootecnia, 2004. 42p.

PARANHOS DA COSTA, M.J.R.; CROMBERG, V.U.; ARDESH, J.H. Diferenças na latência da primeira mamada em quatro raças de bovinos de corte. In: CONGRESSO DE ZOOTECNIA, 6., 1996, Évora. Actas... Evora: Associação Portuguesa de Engenheiros Zootecnicos, 1996. v.2, p.343-348.

RILEY, D.G.; CHASE JR., C.C.; OLSON, T.A. et al. Genetic and nongenetic influences on vigor at birth and preweaning mortality of purebred and high percentage Brahman calves. Journal of Animal Science, v.82, p.1581-1588, 2004.

STATISTICAL ANALYSIS SYSTEM - SAS. [2001]. SAS/STAT user's guide. The GENMOD procedure. Disponível em: <http:SASOnlineDocV8/sasdoc/sashtml/stat/chap30/index.htm> Acesso em: 20/4/2001.

SCHMIDEK, A.; PARANHOS DA COSTA, M.J.R.; MERCADANTE, M.E.Z. et al. The effect of newborn calves vigour in their mortality probability. In: CONGRESS OF INTERNATIONAL SOCIETY OF APPLIED ETHOLOGY, 40., 2006, Bristol. Proceedings... Bristol: Society of Applied Ethology, 2006. p.221.

VENTROP, M.; MICHANEK, P. The importance of udder and teat conformation for teat seeking by the newborn calf. Journal of Dairy Science, v.75, p.262-268, 1992. 